\title{
Determination of the Radio Transmitter Coordinates In Satellite Communication Systems
}

\author{
A. S. Kamyshnikova \\ Institute of engineering physic and radio electronics, \\ Siberian federal university \\ Krasnoyarsk, Russian Federation \\ e-mail: komenzo@yandex.ru
}

\author{
V. V. Sukhotin \\ Institute of engineering physic and radio electronics, \\ Siberian federal university \\ Krasnoyarsk, Russian Federation \\ e-mail: vsuhotin@sfu-kras.ru
}

\begin{abstract}
The method to determine the coordinates of the radio transmitter, which is located outside the Earth's surface, using the virtual antenna array, is described in this article. Variations of the calculation method, based on the construction of the pyramid, are considered. The errors of this method are given.
\end{abstract}

Keywords - satellite communication system, determination of the coordinates, virtual antenna array, radio source, radio transmitter.

\section{INTRODUCTION}

The modern world can not be imagined without satellite technology. Satellite communication systems are widely used in television, telephony, geolocation, etc. Usually satellite communication systems consist of spacecraft used as retranslators and terrestrial or near-Earth transceivers.

Due to the availability of these systems, there may be cases of illegitimate use of spacecraft's frequency resources. [1-3] The various methods based on determining the origin of the radio transmitter are a promising direction in this area. [4-7].

\section{EXISTING METHODS BASED ON THE USE OF THE VIRTUAL ANTENNA ARRAY}

\section{A. Radio Transmitter Is Located on the Earth's Surface}

The method based on the use of the virtual antenna array (further - VAA) is known. Geostationary artificial satellite is not static while the coordinates of its position are known at each instant. Using several sequent satellite positions forms the virtual antenna array. The coordinates of the RT can be determined by measuring the phase difference between the signals emitted by them in these positions of the satellite. [6-7] However, this method does not allow to determine the coordinates of the RT in case if it is unknown whether the RT is located on the Earth's surface or outside it.

\section{B. Method Based on the Use of Cons}

Therefore it is proposed to use an improved variation of the method based on the use of the VAA, which allows to determine the coordinates of the RT which is located outside the Earth's surface.
The method based on the use of cones is described in the paper [7]. With high accuracy it can be assumed that emitting of the antenna on board of the satellite forms in space a circular conical surface, the apex of which is the subsatellite point. Displacement of the satellite leads to the second cone and the associated local coordinate system. The RT is located at the intersection of three conical surfaces. To determine its location, it is necessary to solve a system of equations:

$$
\begin{aligned}
& n_{1}^{1} x^{2}+n_{2}^{1} y^{2}+n_{3}^{1} z^{2}+m_{1}^{1} x y+m_{2}^{1} y z+m_{3}^{1} x z+p_{1}^{1} x+p_{2}^{1} y+p_{3}^{1} z+q_{1}^{1}+q_{2}^{1}+q_{3}^{1}=0 \\
& \left.n_{1}^{2} x^{2}+n_{2}^{2} y^{2}+n_{3}^{2} z^{2}+m_{1}^{2} x y+m_{2}^{2} y z+m_{3}^{2} x z+p_{1}^{2} x+p_{2}^{2} y+p_{3}^{2} z+q_{1}^{2}+q_{2}^{2}+q_{3}^{2}=0\right\} \\
& n_{1}^{1} x^{3}+n_{2}^{1} y^{3}+n_{3}^{1} z^{3}+m_{1}^{3} x y+m_{2}^{3} y z+m_{3}^{3} x z+p_{1}^{3} x+p_{2}^{3} y+p_{3}^{3} z+q_{1}^{3}+q_{2}^{3}+q_{3}^{3}=0 \\
& \text { where } n_{j}^{i}, m_{j}^{i}, p_{j}^{i}, q_{j}^{i} \text { are known numeric coefficients. [8]. }
\end{aligned}
$$

\section{Method BASED ON THE USE OF THE PYRAMID}

However, the disadvantage of the described method is that the solution of such a second-order system of equations requires a lot of computing power. Therefore a simpler way to calculate the coordinates of the RT, which is located outside the Earth's surface, was developed - a method based on the use of the pyramid.

Generally, points $A_{1}, A_{2}, A_{3}$ and $B$ (where the RT is located) define a pyramid wherein the triangle $\mathrm{A}_{1} \mathrm{~A}_{2} \mathrm{~A}_{3}$ is its base and the point $\mathrm{B}$ is its apex as it is shown in Fig. 1.

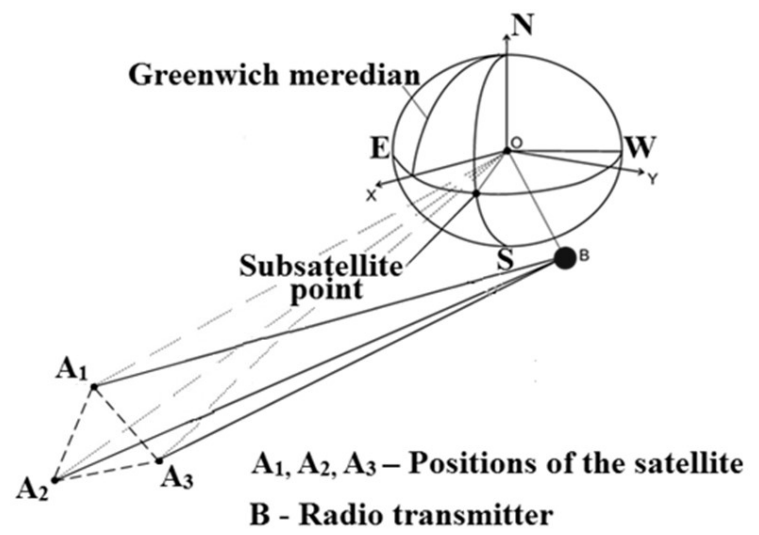

Fig. 1 - General geometrical constructions for the method to determine the radio transmitter coordinates based on the use of the pyramid 
Depending on the relative position of the point B and the base of the pyramid, two cases can be distinguished:

\section{A. Location of the radio transmitter can be projected on the base of the pyramid}

If the RT is located relatively to the satellite so that projection of the point $\mathrm{B}$ on a plane, which is defined by three positions of the satellite (points $A_{1}, A_{2}, A_{3}$ ), is in the limits of the triangle $A_{1} A_{2} A_{3}$, it is expedient to use the following method to determine the RT coordinates.

Define an auxiliary rectangular Cartesian coordinate system (CS) with an origin at the point $\mathrm{A}_{1} ; \mathrm{A}_{1} \mathrm{~A}_{2}$ is $\mathrm{OX}$; the axis $\mathrm{OZ}$ is parallel to the height $\mathrm{BO}$, lowered from the point $\mathrm{B}$ on the base of the pyramid; the axis $\mathrm{OY}$ is perpendicular to axes $\mathrm{OX}$ and OZ (Fig. 2).

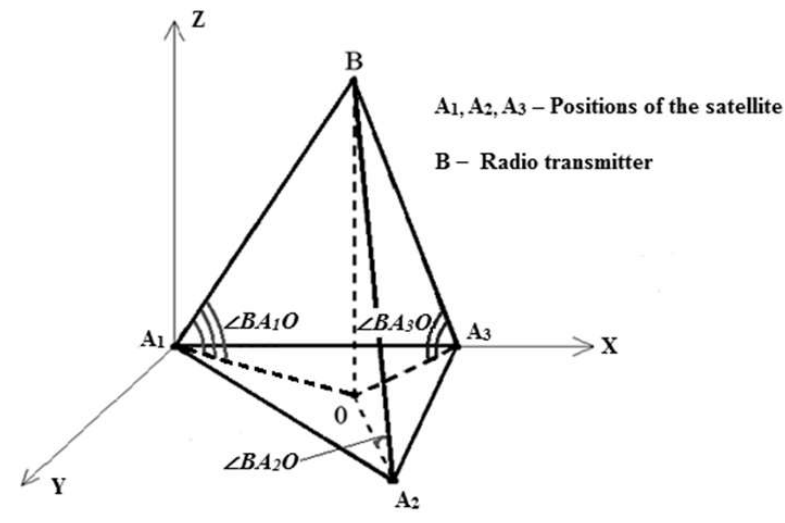

Fig. 2 - An auxiliary coordinate system for the method of determining the radio transmitter coordinates based on the use of the pyramid

In the geocentric $C S$, points $A_{1}, A_{2}, A_{3}$ have following coordinates (satellite ephemeris): $\mathrm{A}_{1}\left(\mathrm{x}_{1} ; \mathrm{y}_{1} ; \mathrm{z}_{1}\right), \mathrm{A}_{2}\left(\mathrm{x}_{2} ; \mathrm{y}_{2} ; \mathrm{z}_{2}\right)$ and $\mathrm{A}_{3}\left(\mathrm{x}_{3} ; \mathrm{y}_{3} ; \mathrm{z}_{3}\right)$.

Determine the angles in the base of the pyramid:

$$
\begin{aligned}
& \angle B A_{1} O=\frac{\pi}{2}-\frac{\psi_{1}}{2} \\
& \angle B A_{2} O=\frac{\pi}{2}-\frac{\psi_{2}}{2} \\
& \angle B A_{3} O=\frac{\pi}{2}-\frac{\psi_{3}}{2}
\end{aligned}
$$

where $\psi_{1}, \psi_{2}, \psi_{3}$ are the angles of the axial section of the conical surfaces.

Knowing the side lengths of the base of the pyramid (it follows from the fact that coordinates of the points $A_{1}, A_{2}, A_{3}$ are known) and the angles (2), we are able to calculate the coordinates of the point B in the auxiliary CS. To calculate the RT coordinates in the geocentric CS, we must execute the axis rotation of the auxiliary CS and its parallel transport.
B. Location of the radio transmitter cannot be projected on the base of the pyramid

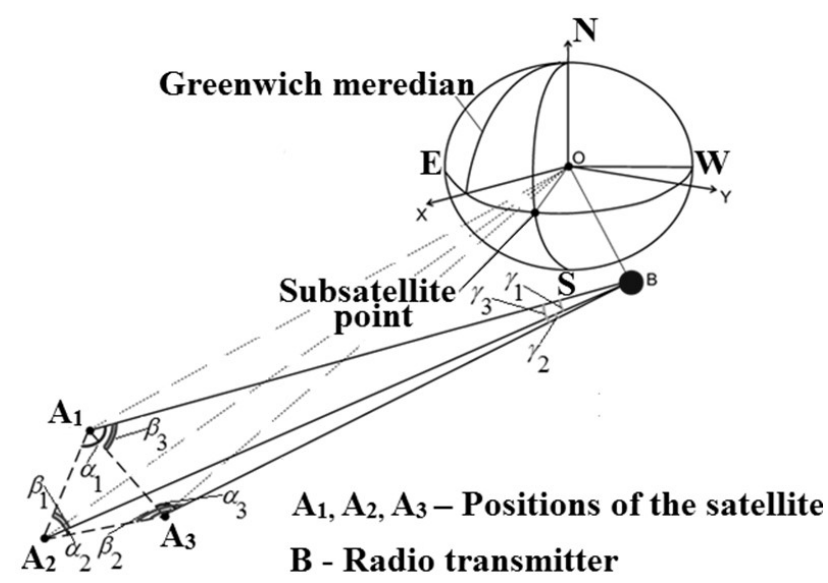

Fig. 3 - Geometric constructions for the method of determining the radio transmitter coordinates based on the use of the pyramid

The angles in the bases of each of the faces of the pyramid are determined based on the phase difference of the incoming signal at the points $\mathrm{A}_{1}, \mathrm{~A}_{2}, \mathrm{~A}_{3}$ :

$$
\begin{aligned}
& \alpha_{i}=\arccos \left(\frac{\lambda}{2 \pi d_{i}} \cdot \Delta \psi_{i j}\right), \\
& \beta_{i}=\arccos \left(\frac{\lambda}{2 \pi d_{i}} \cdot \Delta \psi_{j i}\right),
\end{aligned}
$$

where di is the ith base of VAA,

$\Delta \psi_{\mathrm{ij}}$ is the phase difference of the incoming signal to the adjacent points. [9]

Knowing these angles and the coordinates of the points in base of the pyramid, we are able to build a system of equations based on the Law of Sines:

$$
\begin{aligned}
& \sqrt{\left(x_{2}-x_{B}\right)^{2}+\left(y_{2}-y_{B}\right)^{2}+\left(\mathrm{z}_{2}-\mathrm{z}_{B}\right)^{2}} \cdot \sin \gamma_{1}=\sin \alpha_{1} \cdot A_{1} A_{2} \\
& \sqrt{\left(x_{3}-x_{B}\right)^{2}+\left(y_{3}-y_{B}\right)^{2}+\left(\mathrm{z}_{3}-\mathrm{z}_{B}\right)^{2}} \cdot \sin \gamma_{2}=\sin \alpha_{2} \cdot A_{2} A_{3}, \\
& \sqrt{\left(x_{1}-x_{B}\right)^{2}+\left(y_{1}-y_{B}\right)^{2}+\left(\mathrm{z}_{1}-\mathrm{z}_{B}\right)^{2}} \cdot \sin \gamma_{3}=\sin \alpha_{3} \cdot A_{3} A_{1}
\end{aligned}
$$

where $\mathrm{x}_{1}, \mathrm{y}_{1}, \mathrm{z}_{1}$ are the coordinates of the point $\mathrm{A}_{1}$,

$\mathrm{x}_{2}, \mathrm{y}_{2}, \mathrm{z}_{2}$ are the coordinates of the point $\mathrm{A}_{2}$,

$\mathrm{x}_{3}, \mathrm{y}_{3}, \mathrm{z}_{3}$ are the coordinates of the point $\mathrm{A}_{3}$,

$\mathrm{X}_{\mathrm{B}}, \mathrm{y}_{\mathrm{B}}, \mathrm{Z}_{\mathrm{B}}$ are the coordinates of the point $\mathrm{B}$.

To calculate the coordinates of the point B, i.e. the RT coordinates, we must solve this system.

Comparing the two above-described methods, we come to the following conclusions: the first method is simpler mathematically, but is suitable for a limited number of cases. 
The second method is more general, however, the calculations are more complex.

\section{ERrors OF THE METHOD BASED ON THE USE OF THE PYRAMID}

Ones of the main sources of errors are the inaccuracies of the satellite ephemerides prediction and the error of the phase difference measurement.

\section{A. Impact of the inaccuracies of the satellite ephemerides prediction}

There are a complex dependences between the errors of determining the RT coordinates and the inaccuracies of the satellite ephemerides prediction. When changing the satellite ephemerides prediction inaccuracy from 0 to $100 \mathrm{~m}$ by the ephemeris $\mathrm{X}_{2}$, resultant error of determining the RT coordinates ranges from 5 to $50 \mathrm{~m}$ as it is shown in Fig. 4. [10]. a)



c)

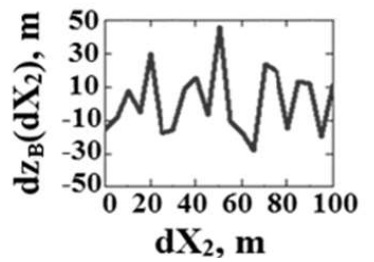

b)

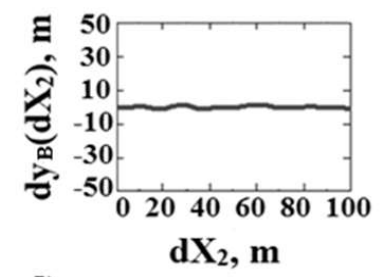

d)

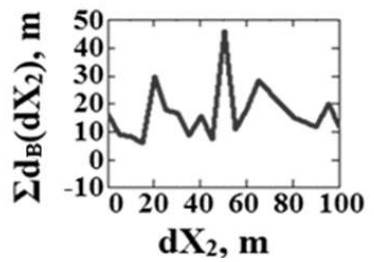

Fig. 4 - Dependences between the errors of determining the radio transmitter coordinates and the inaccuracy of the satellite ephemeris prediction, $\mathrm{dX}_{2}$, at the point $\mathrm{A}_{2}$ of the virtual antenna array:

$a$ - Error of determining the radio transmitter coordinate $\mathrm{X}_{\mathrm{B}}$

$\mathrm{b}-$ Error of determining the radio transmitter coordinate $\mathrm{y}_{\mathrm{B}}$

$\mathrm{c}-$ Error of determining the radio transmitter coordinate $\mathrm{Z}_{\mathrm{B}}$

$\mathrm{d}$ - Resultant error of determining the radio transmitter coordinates

Plots of dependences between the errors of determining the RT coordinates and the inaccuracies of the satellite ephemerides prediction by the ephemerides $Y_{2}$ and $Z_{2}$ are similar to those shown. [10].

\section{B. Impact of the error of the phase difference measurement}

There are strictly increasing dependences between the errors of determining the RT coordinates and the error of the phase difference measurement. When changing the error of the phase difference measurement $\mathrm{d} \psi$ from $0^{\circ}$ to $1^{\circ}$, resultant error of determining the RT coordinates ranges from 0 to $2000 \mathrm{~km}$ as it is shown in Fig. 5. [10]. a)

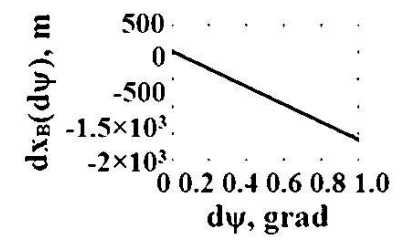

c)

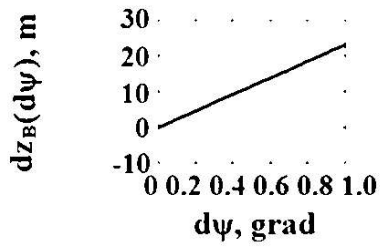

b)

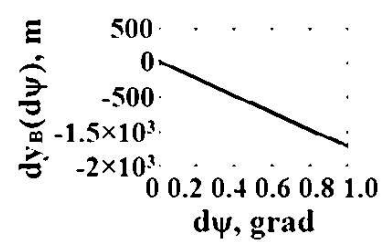

d)

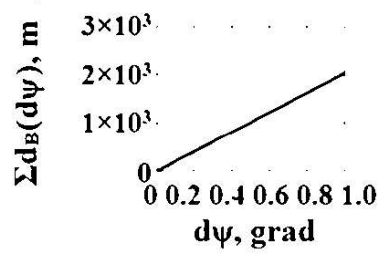

Fig. 5 - Dependences between the errors of determining the radio transmitter coordinates and the error of the phase difference measurement, $d \psi$ :

$a-$ Error of determining the radio transmitter coordinate $\mathrm{X}_{\mathrm{B}}$

$\mathrm{b}-$ Error of determining the radio transmitter coordinate ув $_{\mathrm{B}}$

$\mathrm{c}-$ Error of determining the radio transmitter coordinate $\mathrm{Z}_{\mathrm{B}}$

$\mathrm{d}$ - Resultant error of determining the radio transmitter coordinates

Therefore, the method described above allows to determine the coordinates of the RT which is located outside the Earth's surface, that makes it possible to extend the applicability of the method based on the use of the VAA. However, based on the error study above, it is possible to draw a conclusion about the need to pay more attention to the implementation of the phase meter, since its error is the largest contributor to the errors of determining the RT coordinates.

\section{REFERENCES}

[1] Haworth D. P., Smith N. G., Bardelli R., Clement T. Interference localization for Eutelsat satellites - the first European transmitter location system, International Journal of satellite communications, Vol. 15, 1997, P. 155-183.

[2] Panko S.P., Sukhotin V.V. Unauthorized access to the satellite communication systems. Electronics Overseas. Achievements of modern Radioelectronics. - M.: Radiotechnics, № 4, 2002.

[3] C. Privat, L. Sterenberg. Interference Detection and Geolocation System, Technical Proposals, Alcatel Alenia Space, 2005.

[4] Moguchev V.I. Doppler direction findings of terrestrial stations via geostationary communication satellite. - M.: Telecommunications, №1, 2003.

[5] Patent RU2450284 (MPK2), G01S5/02 - Method of vsat-positioning in satellite network / Voronin A.V., Ivanov V.N., Academy of the Federal Protective Service; Application number: RU2010121505/07 20010608 2012.

[6] Kalashnikova A.S., Sukhotin V.V. Methods to protect frequency resources of satellite system. Modern problems of radio electronics : Proceedings [Electronic resource] / Science editor S. P. Panko ; responsible for issue A. A. Levitskiy. - Electronic data (32 Mb). Krasnoyarsk : Sibirean State University, 2014. - 606 p.

[7] Kalashnikova A.S., Sukhotin V.V. Consideration of methods to protect frequency resources of satellite system against unauthorized access. 2015 International Siberian Conference on Control and Communications (SIBCON). Proceedings. - Omsk: Omsk State Technical University. Russia, Omsk, May 21-23, 2015. IEEE Catalog Number: CFP15794CDR. ISBN: 978-1-4799-7102-2. 
[8] Ilin B.A., Poznyak E.G. Analytic geometry. - M.: Fizmalit, 2004. - 224 $p$

[9] R.M. Wachs. Communications satellite interference location system. US Patent №6147640, 2000.
[10] Kalashnikova A.S., Sukhotin V.V., Admaev O.V., Smolnikov E.O. Method to determine the coordinates of the radio transmitter using a geostationary artificial satellite. Achievements of Modern Radioelectronics. - M.: Radiotechnics, №10, 2015 